\title{
APLICAÇÃO DA METODOLOGIA DE SUPERFÍCIE DE RESPOSTA DURANTE A SECAGEM DE SEMENTES DE PAINÇO EM LEITO DE JORRO
}

\author{
L. D. NASCIMENTO ${ }^{1 *}$, S. C. S. ROCHA ${ }^{2}$, O. P.TARANTO ${ }^{2}$, C.M.L. COSTA ${ }^{3}$, L.J.G. FARIA ${ }^{3}$ \\ ${ }^{1}$ Universidade Federal do Pará, Programa de Pós Graduação em Engenharia Química; \\ Museu Paraense Emílio Goeldi \\ ${ }^{2}$ Universidade Estadual de Campinas, Faculdade de Engenharia Química \\ ${ }^{3}$ Universidade Federal do Pará, Faculdade de Engenharia Química. \\ e-mail: lidiane1610@gmail.com
}

\begin{abstract}
RESUMO
A secagem de sementes é importante para a obtenção de um produto final em condições de ser armazenado, comercializado ou até mesmo processado e o leito de jorro é uma alternativa para a realização de operações de secagem, assim como para o recobrimento de partículas e desenvolvimento de reações. As partículas utilizadas neste trabalho foram sementes de painço, as quais podem se tornar uma possível alternativa na alimentação de diabéticos. O objetivo deste trabalho é verificar como a temperatura e o tempo de secagem influenciam na resposta razão de umidade, por meio de um modelo matemático, durante a secagem de sementes de painço em leito de jorro. Elaborou-se um planejamento composto central rotacional com o objetivo de avaliar a influência das variáveis independentes tempo $\left(\mathrm{X}_{1}\right)$ e temperatura $\left(\mathrm{X}_{2}\right)$ na resposta razão de umidade $\left(X_{R}\right)$. Para um intervalo de confiança de $95 \%$, verificou-se que a variável de resposta $X_{R}$ sofreu influência apenas de $\mathrm{X}_{1}, \mathrm{X}_{2}$ e $\mathrm{X}_{2}^{2}$, sendo proposto um modelo com $\mathrm{R}^{2}$ igual a $96,32 \%$. O leito de jorro foi eficaz para a secagem de sementes de painço e por meio da superfície de resposta e curvas de nível, observou-se que os menores valores para a razão de umidade $\left(\mathrm{X}_{\mathrm{R}}\right)$ são obtidos quando opera-se em elevadas temperaturas em qualquer um dos intervalos de tempo analisados.
\end{abstract}

\section{INTRODUÇÃO}

O leito de jorro tem se mostrado uma técnica versátil, sendo utilizado para a secagem de sementes (NASCIMENTO et al., 2014), polpas (ARAUJO et al., 2015), recobrimento de partículas (CHEN; KUO, 2015) e até mesmo como reator (SAN JOSÉ et al., 2014; MAKIBAR et al., 2015).

De acordo com Mujumdar (2006) a secagem de sólidos é uma das mais antigas e usuais operações unitárias encontradas nas indústrias agrícola, cerâmica, química, alimentícia, entre outras. Neste processo, ocorrem simultaneamente dois fenômenos: transferência de energia (calor) do ambiente para evaporar a umidade superficial e transferência de massa (umidade), do interior para a superfície do material e sua subsequente evaporação devido ao primeiro processo (PARK, 2007). Secadores do tipo leito de jorro são apropriados para partículas pertencentes ao Grupo D (como por exemplo as sementes de painço) na classificação de Geldart, as quais são grosseiras e densas demais para um leito fluidizado convencional. Neste sistema a movimentação das partículas é regular e circular (YANG, 
2003) o que favorece as taxas de transferência de calor em massa.

No Brasil, as sementes de painço ( $S$. italica) são destinadas principalmente para a alimentação de pássaros (CORRÊA et al., 2006), mas são utilizadas na alimentação humana em países como China, Índia e Rússia. De acordo com os estudos de Anju e Sarita (2010), as sementes podem ser utilizadas como alternativa na alimentação de diabéticos, pois cookies preparados a partir da farinha dessas sementes apresentaram menores índices glicêmicos, quando comparados àqueles feitos apenas com farinha de trigo.

A Metodologia de Superfície de Resposta é uma técnica de otimização baseada em planejamentos fatoriais que foi introduzida por G.E.P. Box na década de 1950 (BARROS NETO; SCARMINIO; BRUNS, 2010), a qual explora relações existentes entre algumas variáveis que supostamente interferem no processo (conhecidas como variáveis de entrada) com uma variável resposta (conhecida como variável dependente), tal como o teor umidade final de uma semente.

Este trabalho utiliza o leito de jorro como uma alternativa para a secagem de sementes de painço e verifica por meio de um planejamento de experimentos como a temperatura e o tempo de secagem podem influenciar a resposta razão de umidade, propondo então um modelo matemático que relacione estas variáveis.

\section{MATERIAL E MÉTODOS}

\subsection{Aquisição do Material}

Sementes de $S$. italica foram adquiridas no comércio varejista e armazenadas em um refrigerador a uma temperatura de $6{ }^{\circ} \mathrm{C}$ até a realização dos experimentos.

\subsection{Configuração do Leito de Jorro}

Os ensaios experimentais foram realizados em um leito de jorro com base cônica de $0,15 \mathrm{~m}$ de altura, ângulo de $60^{\circ} \mathrm{e}$ diâmetro de entrada do ar $\left(\mathrm{D}_{\mathrm{i}}\right)$ de $0,0254 \mathrm{~m}$. A coluna cilíndrica apresentava $0,8 \mathrm{~m}$ de altura e diâmetro interno (Dc) de $0,16 \mathrm{~m}$, de tal forma que a relação Di/Dc foi de 0,16 .

\subsection{Umidificação}

Uma vez que as sementes adquiridas apresentavam um teor de umidade em base seca $13,85 \% \pm 1,02$, foi necessário umidificálas para dar prosseguimento aos ensaios de secagem. Para isso, foi realizado o seguinte procedimento: aspergiu-se um volume definido de água destilada sobre as sementes, a cada $24 \mathrm{~h}$, completando um ciclo de $72 \mathrm{~h}$ a fim de alcançar um teor de umidade em média igual a $30 \% \pm 0,01 \%$ em base seca. Ao término de cada aspersão, as sementes eram armazenadas à $6^{\circ} \mathrm{C}$ até a realização dos experimentos de secagem.

\subsection{Delineamento Experimental}

Foram utilizados cinco níveis de tempo: $48,60,90,120$ e 132 min e cinco diferentes temperaturas: $44,50,65,80$ e $86^{\circ} \mathrm{C}$ conforme a Tabela 1. O projeto experimental consistiu em um planejamento composto central rotacional (PCCR), com quatro repetições no ponto central, totalizando 12 ensaios, para determinar a influência dos parâmetros tempo $\left(\mathrm{X}_{1}\right)$ e temperatura do ar de secagem $\left(\mathrm{X}_{2}\right)$ sobre a resposta razão de umidade $\left(\mathrm{X}_{\mathrm{R}}\right)$, definida conforme a Equação 1. X(t), Xi e Xe representam o teor de umidade da amostra em função do tempo, a umidade inicial da amostra e a umidade de equilíbrio, respectivamente.

$$
X_{R}=\frac{X(t)-X_{\theta}}{X_{\mathrm{i}}-X_{\theta}}=\frac{X(t)}{X_{\mathrm{i}}}
$$


A interpretação dos resultados foi realizada utilizando o aplicativo Statistica ${ }^{\circledR} 7.0$ e a metodologia de superfície de resposta (em função do modelo proposto para a variável de resposta). Foi considerado um nível de significância $\alpha=0,05$ (confiança 95\%).

Tabela 1 - Valores codificados e originais para as variáveis utilizadas no PCCR.

\begin{tabular}{ccccccc} 
& \multicolumn{5}{c}{ Níveis } \\
\cline { 2 - 6 } $\begin{array}{c}\text { Variáveis codificadas } \\
\text { (originais, unidade) }\end{array}$ & $-1,41$ & -1 & 0 & +1 & $+1,41$ \\
\hline $\begin{array}{c}\mathrm{X}_{1} \text { (tempo de secagem, } \\
\text { minuto) }\end{array}$ & 48 & 60 & 90 & 120 & 132 \\
$\mathrm{X}_{2}\left(\begin{array}{c}\text { (temperatura do } \\
\left.\text { ar, }{ }^{\circ} \mathrm{C}\right)\end{array}\right.$ & 44 & 50 & 65 & 80 & 86
\end{tabular}

\section{RESULTADOS E DISCUSSÃO}

A Tabela 2 mostra a matriz de experimentos composta pelas variáveis avaliadas durante o processo de secagem de sementes de painço em leito de jorro, seguindo um PCCR.

Tabela 2 - Matriz de experimentos e os resultados experimentais para a secagem das sementes de painço em leito de jorro.

\begin{tabular}{cccc}
\hline \multirow{2}{*}{ Corridas } & \multicolumn{2}{c}{$\begin{array}{c}\text { Variáveis } \\
\text { Codificadas }\end{array}$} & $\begin{array}{c}\text { Variável de } \\
\text { Resposta }\end{array}$ \\
\cline { 2 - 4 } & $\mathrm{X}_{1}$ & $\mathrm{X}_{2}$ & $\begin{array}{c}\mathrm{X}_{\mathrm{R}} \\
\text { adim.) }\end{array}$ \\
\hline 1 & -1 & -1 & 0,67 \\
2 & +1 & -1 & 0,59 \\
3 & -1 & +1 & 0,44 \\
4 & +1 & +1 & 0,28 \\
5 & $-1,41$ & 0 & 0,53 \\
6 & $+1,41$ & 0 & 0,33 \\
7 & 0 & $-1,41$ & 0,76 \\
8 & 0 & $+1,41$ & 0,35 \\
9 & 0 & 0 & 0,35 \\
10 & 0 & 0 & 0,4 \\
11 & 0 & 0 & 0,43 \\
12 & 0 & 0 & 0,45
\end{tabular}

Os valores de teor de umidade final das sementes submetidas a secagem em leito de jorro, ficaram na faixa entre 18,35 e 9,32\% em base seca, mostrando que é possível obter sementes com teores de umidade apropriados para uma armazenagem livre do ataque de insetos (SOAVE; MORAES, 1987).

\subsection{Análise de variância (ANOVA)}

A análise de variância permite avaliar a probabilidade que cada efeito possui de ser considerado estatisticamente significativo para a variável de resposta. Portanto, foi realizada a ANOVA para a resposta $X_{R}$ e de acordo com os resultados obtidos para um nível de $95 \%$ de confiança, somente as variáveis $X_{1}, X_{2}$ e $X_{2}^{2}$ foram estatisticamente significativas, pois seus valores de $p$ apresentaram menor probabilidade de estar dentro da região de hipótese nula, o que foi evidenciado pela ordem de grandeza dos valores numéricos encontrados. Em seguida foi proposto um modelo completo para a variável de resposta razão de umidade, como mostra a Equação 2, em função das variáveis de entrada, cujos efeitos e coeficientes de regressão estão sumarizados na Tabela 3. A partir desta Tabela, é verificado que os efeitos provocados pelas variáveis codificadas $\mathrm{X}_{1} \mathrm{e}$ $\mathrm{X}_{2}$ são negativos, portanto a elevação dos valores de temperatura de $44^{\circ} \mathrm{C}$ para $86^{\circ} \mathrm{C}$ e o aumento do tempo de $48 \mathrm{~min}$ para $132 \mathrm{~min}$, provocam efeitos negativos na resposta $X_{R}$, ou seja, maiores valores de temperatura e tempo de secagem favorecem a redução do teor de umidade final das sementes. 
Tabela 3 - Efeitos e coeficientes de regressão para a resposta razão de umidade.

\begin{tabular}{ccc}
\hline Fonte de variação & Efeito & $\begin{array}{c}\text { Coeficientes de } \\
\text { regressão } \\
\text { estimados } \pm \text { erro }\end{array}$ \\
\hline Grande média & $0,41 \pm 0,02$ & $0,41 \pm 0,02$ \\
$\mathrm{X}_{1}$ & $-0,13 \pm 0,03$ & $-0,06 \pm 0,02$ \\
$\mathrm{X}_{1}{ }^{2}$ & $0,024 \pm 0,03$ & $0,01 \pm 0,02$ \\
$\mathrm{X}_{2}$ & $-0,28 \pm 0,03$ & $-0,14 \pm 0,02$ \\
$\mathrm{X}_{2}{ }^{2}$ & $0,15 \pm 0,03$ & $0,07 \pm 0,02$ \\
$\mathrm{X}_{1} \mathrm{X}_{2}$ & $-0,04 \pm 0,04$ & $-0,02 \pm 0,02$ \\
& & \\
$X_{R}=0,4075-0,0654 X_{1}-$ & \\
$0,1402 X_{2}-0,02 X_{1} X_{2}+$ & \\
$0,0118 X_{1}^{2}+0,0747 X_{2}^{2}$ &
\end{tabular}

Onde as variáveis codificadas estão relacionadas às variáveis originais conforme as Equações 3 e 4:

$$
\begin{aligned}
& X_{1}=\frac{t-90}{30} \\
& X_{2}=\frac{T-65}{15}
\end{aligned}
$$

O modelo descrito pela Equação 2 representou bem os dados experimentais, de acordo com as restrições deste trabalho, uma vez que seu coeficiente de determinação múltipla $\left(\mathrm{R}^{2}\right)$ foi equivalente à 0,9738 . No entanto, a fim de verificar a possibilidade de reduzir os parâmetros na equação para a resposta $X_{R}$ (Equação 2), foi realizada uma nova análise de variância, como mostra a Tabela 4, considerando apenas as variáveis que apresentaram significância estatística, ou seja, $X_{1}, X_{2}$ e $X_{2}^{2}$ e obteve-se o modelo reduzido, como mostra a Equação 5.
Tabela 4 - ANOVA para a variável de resposta $\mathrm{X}_{\mathrm{R}}$ considerando apenas as variáveis com significância estatística.

\begin{tabular}{ccccc}
\hline $\begin{array}{c}\text { Fonte de } \\
\text { variação }\end{array}$ & $\begin{array}{c}\text { Soma dos } \\
\text { quadrados }\end{array}$ & $\begin{array}{c}\text { Graus de } \\
\text { liberdade }\end{array}$ & $\begin{array}{c}\text { Quadrado } \\
\text { médio }\end{array}$ & $p$ \\
\hline $\mathrm{X}_{1}$ & 0,03 & 1 & 0,03 & 0,02 \\
$\mathrm{X}_{2}$ & 0,15 & 1 & 0,15 & 0,00 \\
$\mathrm{X}_{2}^{2}$ & 0,03 & 1 & 0,03 & 0,02 \\
$\begin{array}{c}\text { Falta de } \\
\text { ajuste }\end{array}$ & 0,00 & 5 & 0,00 & 0,87 \\
$\begin{array}{c}\text { Erro puro } \\
\text { Total }\end{array}$ & 0,00 & 3 & 0,00 & - \\
$\begin{array}{c}\text { correlação) } \\
\mathrm{R}^{2}=0,9632\end{array}$ & 0,23 & 11 & - & - \\
\\
$X_{R}=0,05$. \\
$0,1402 X_{2}+0,0747 X_{2}^{2}$
\end{tabular}

De acordo com a Tabela 4, foi verificado que não houve alteração na influência das variáveis $\mathrm{X}_{1}, \mathrm{X}_{2}$ e $\mathrm{X}_{2}^{2}$ sobre a resposta $X_{R}$ e que o modelo reduzido (Equação 5) conseguiu reproduzir ou explicar $96,32 \%$ das variabilidades experimentais, valor inferior ao alcançado pelo modelo completo, mas ainda muito satisfatório. Com base nos modelos completo e reduzido, é possível verificar a influência quadrática da temperatura e linear do tempo na resposta $\mathrm{X}_{\mathrm{R}}$.

A Figura 1 ilustra a correlação entre os valores preditos pelo modelo reduzido e os valores observados experimentalmente, no qual verifica-se que a maioria dos resíduos estão localizados próximo ao zero e com distribuição em torno da curva normal, indicando um bom ajuste ao modelo proposto. 
Figura 1 - Gráfico normal dos resíduos para $X_{R}$ em função do modelo reduzido.

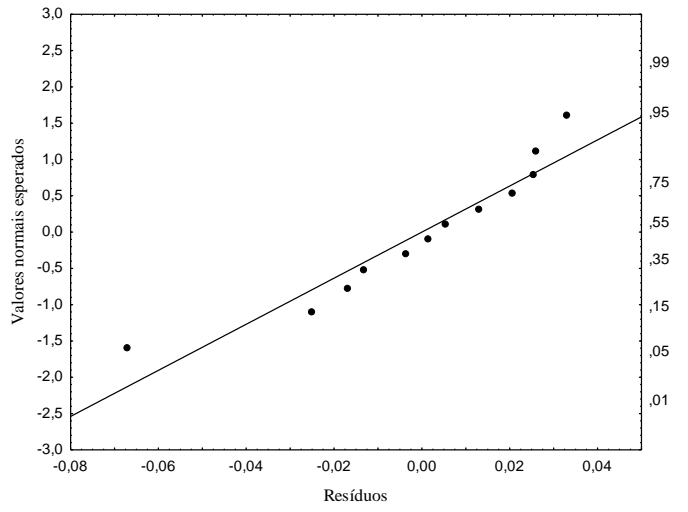

\subsection{Superfície de resposta e curvas de nível para $X_{R}$}

O modelo estatístico reduzido proposto para $\mathrm{X}_{\mathrm{R}}$ é visualizado por meio da superfície de resposta e respectivas curvas de nível, conforme as Figuras 2 e 3, respectivamente. Representa-se no eixo $\mathrm{z}$ a resposta, neste caso $\mathrm{X}_{\mathrm{R}}$, e nos eixos $\mathrm{x}$ e $\mathrm{y}$ as duas variáveis independentes $\mathrm{X}_{1}$ e $\mathrm{X}_{2}$. É importante ressaltar que os valores nos eixos $x$ e $y$ estão codificados, portanto os mesmos precisam ser inseridos nas Equações 3 e 4 para obter os valores reais.

Verifica-se que para a obtenção de baixos valores de razão de umidade $\left(X_{R}\right)$ é necessário operar em altas temperaturas (elevados níveis de $\mathrm{X}_{2}$ ) em qualquer intervalo de tempo $\left(\mathrm{X}_{1}\right)$ avaliado, sobretudo nos superiores.

Figura 2 - Superfície de resposta para $X_{R}$.

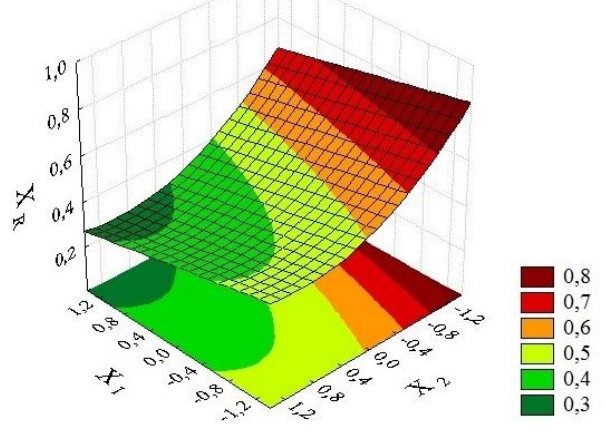

Figura 3 - Curvas de nível para $X_{R}$.

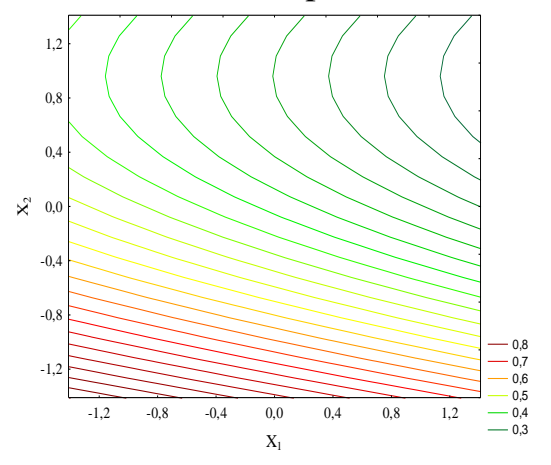

\section{CONCLUSÃO}

O leito de jorro se mostrou uma alternativa eficaz para a secagem de sementes de painço, de maneira que por meio das análises realizadas a partir da superfície de resposta e curvas de nível, os menores valores para a razão de umidade (XR) são obtidos quando opera-se em elevadas temperaturas e qualquer um dos intervalos de tempo analisados, sobretudo os maiores tempos de operação.

As influências estatisticamente significativas na resposta $X_{R}$, foram $X_{1}, X_{2}$ e $\mathrm{X}_{2}^{2}$, ambas influenciando na redução da razão de umidade, ou seja, reduzindo o teor de umidade final das sementes. Portanto, o modelo proposto mostra a influência quadrática da temperatura e linear do tempo na razão de umidade durante a secagem de sementes de painço em leito de jorro.

\section{REFERÊNCIAS}

ANJU, T.; SARITA, S. Suitability of Foxtail Millet (Setaria itálica) and Barnyard Millet ( Echinochloa frumentacea) for development of low glycemic index biscuits. Malaysian Journal of Nutrition, v.16, p. $361-368$. 2010.

ARAÚJO, A.D.A; COELHO, R.M.D.; FONTES, C.P.M.L.; SILVA, A.R.A.; DA COSTA, J.M.C; RODRIGUES, S. Production 
and spouted bed drying of acerola juicecontaining oligosaccharides. Food and Bioproducts Processing, v. 9 4, p. 565-571, 2015.

BARROS NETO, B.; SCARMINIO, I.S.; BRUNS, R.E. Como fazer experimentos: pesquisa e desenvolvimento na ciência e na indústria. $4^{\mathrm{a}}$ Edição. Porto Alegre: Bookman, 2010.

CHEN, W.Y.; KUO, H.P. Surface Coating of Group B Iron Powders in a Spouted Bed. Procedia Engineering, v. 102, p. 1144 1149, 2015.

CORRÊA, P.C.; JÚNIOR, P.C.A.; RIBEIRO, D.M.; SILVA, F.F. Equilíbrio higroscópico de milheto, alpiste e painço: Obtenção e modelagem. Revista Brasileira de Engenharia Agrícola e Ambiental, v.10, n.1, p.162-167, 2006.

NASCIMENTO, L. D.; CORUMBA, L. G.; ROCHA, S. C. S.; TARANTO, O. P.; COSTA, C.M.L.; FARIA, L. J. G. Mathematical modeling of the drying curves of foxtail millet seeds in spouted bed. In: 15th Brazilian Congress of Thermal Sciences and Engineering, 2014, Belém. Proceedings..., 2014.

MAKIBAR, J.; FERNANDEZ-AKARREGI, A.R.; AMUTIO , M.; LOPEZ, G.; OLAZAR, M. Performance of a conical spouted bed pilot plant for bio-oil production by poplar flash pyrolysis. Fuel Processing Technology, v. 137, p. 283-289, 2015.

MUJUMDAR, A.S. Handbook of Industrial Drying. Third Edition. New York: Taylor \& Francis Group, 2006.

PARK, K.J.; ANTONIO, G.C.; OLIVEIRA, R.A.; PARK, K.J.B. Conceitos de Processo e Equipamentos de Secagem. 2007.
Disponível

em: <http://www.feagri.unicamp.br/ctea/projpesq. html> Acesso em: 16 fev. 2013.

SAN JOSÉ, M.J.; ALVAREZ, S.; GARCÍA, I., PENAS, F.J. Conical spouted bed combustor for clean valorization of sludge wastes from paper industry to generate energy. Chemical Engineering Research and Design, v. 92, p. 672-678, 2014.

SOAVE J; MORAES SA. Medidas de controle de doenças transmitidas por sementes. In: SOAVE J; WETZEL M.M. (Coord). Patologia de sementes, Campinas, Fundação Cargill, 1987.

YANG, W. C. Handbook of fluidization and fluid particle systems. Nova York: Marcel Dekker, 2003.

\section{AGRADECIMENTOS}

Os autores agradecem ao $\mathrm{CNPq}$ (Processo 552374/2011-3) pelo suporte financeiro. 